\title{
Oxygen Abundances for the Planetary Nebulae in M32, NGC 185, and NGC 205
}

\author{
Michael G. Richer \\ Observatorio Astronómico Nacional, Instituto de Astronomía, UNAM, \\ P.O. Box 439027, San Diego, CA 92143-9027, USA \\ Marshall L. McCall \\ Dept. of Physics \& Astronomy, York University, 4700 Keele St., \\ Toronto, Ontario, Canada M3J 1 P3
}

\begin{abstract}
We present spectroscopy of planetary nebulae in M32, NGC 185 , and NGC 205. With these new data, spectra exist for 14 planetary nebulae in M32, for 5 planetary nebulae in NGC 185, and 13 planetary nebulae in NGC 205. From these data, oxygen abundances based upon electron temperatures derived from detections of $[\mathrm{O}$ III $] \lambda 4363$ may be derived for 11 planetary nebulae in M32, 5 in NGC 185, and 7 in NGC 205. The mean oxygen abundances and their dispersions based upon these [O III] $\lambda 4363$ detections are $8.51 \pm 0.20,8.21 \pm 0.27$, and $8.20 \pm 0.24$ in M32, NGC 185, and NGC 205, respectively, in the usual $12+\log (O / H)$ format.

We confirm a previous finding that a significant fraction of the planetary nebulae in M32 have anomalously strong [N II] lines (Stasińska et al. 1998). Seven of the planetary nebulae for which there now exist spectra (half of the total) have $I([\mathrm{~N} \mathrm{II}] \lambda 6584) / I(\mathrm{H} \beta)>1.5$. These objects are concentrated towards the centre of M32, though less so than the light.

When we combine these new oxygen abundances with extant data, we find that the the same physical processes appear to control the chemical evolution of ellipticals, dwarf spheroidals, and the bulges of spirals. In addition, it is clear that dwarf spheroidals incorporated their metal production into stars much more efficiently than dwarf irregulars, casting more doubt upon an evolutionary link between these two types of dwarf galaxies.
\end{abstract}

\section{References}

Stasińska, G., Richer, M. G., \& McCall, M. L. 1998, A\&A, 336, 667 